\author{
Christian E. Besimo
}

Die interdisziplinäre Therapieplanung bildet die Grundlage einer jeden prothetischen Rehabilitation. An erster Stelle steht die Planung der Initialtherapie mit dem Ziel gesunder und stabiler oraler Verhältnisse als Voraussetzung für die Anfertigung von definitivem Zahnersatz [1-6]. Die zahnärztlichen Interventionen erfolgen in einer zeitlich klar definierten Abfolge und werden mit möglicherweise parallel laufenden medizinischen Interventionen koordiniert, damit die Reaktionen des Organismus auf diese korrekt interpretiert werden können. So liefern die biologischen Antworten auf die durchgeführten Therapiemassnahmen zuverlässige Entscheidungskriterien für die weiter zu planenden Interventionen im Rahmen der Vorbehandlung und für die definitive rekonstruktive Planung. Sie werden jeweils nach Abwarten einer in der Dauer individuell festzulegenden überwachten Heilungsphase evaluiert [7].

Die biologische Antwort umfasst neben der Reaktion der oralen Gewebe auf die Initialtherapie auch Änderungen der Motivation und Mitarbeit des Patienten (Compliance) und der diesem hierzu zur Verfügung stehenden psychischen sowie physischen Ressourcen als wichtige Indikatoren für einen langfristigen Therapieerfolg [8]. Ein weiterer wichtiger Anteil der biologischen Antwort betrifft die Adaptation des Patienten an funktionell bzw. kosmetisch veränderte orale Verhältnisse [9]. Die Initialtherapie

\title{
Nutzung der biologischen Therapieantwort als evidenzbasierte Grundlage einer ganzheitlichen Oralen Medizin
}

Gesunde und stabile orale Verhältnisse sind Voraussetzung einer jeden rekonstruktiven Therapie in der Oralen Medizin und müssen im Rahmen einer Vorbehandlung sichergestellt werden. Die Nutzung der biologischen Antwort auf Interventionen im Rahmen dieser Initialtherapie liefert die Entscheidungskriterien für die definitive rekonstruktive Planung. Diese Behandlungsphase muss bei entsprechender Fragestellung auch Massnahmen umfassen, die die Austestung des angestrebten prothetischen Behandlungsziels vor der definitiven Rekonstruktion unter patienteneigenen biologischen Verhältnissen erlauben. Dabei ist zu gewährleisten, dass bei fehlender Adaptation an die neue Situation die funktionelle Ausgangslage jederzeit wieder hergestellt werden kann. Dieses ganzheitlich wohldefinierte Vorgehen bietet eine hohe biologische und technische Sicherheit.

muss deshalb auch Massnahmen enthalten, die es bei entsprechender Fragestellung erlauben, die prothetische Gesamtplanung vor der definitiven rekonstruktiven Versorgung über längere Zeit unter patienteneigenen biologischen Verhältnissen auszutesten. Hierfür eignen sich Langzeitprovisorien, die in Form und Funktion bereits der angestrebten prothetischen Versorgung entsprechen $[10,11]$. Sofern eine grundlegende Veränderung der funktionellen und kosmetischen Verhältnisse angestrebt wird, sollte durch geeignete diagnostische und therapeutische Massnahmen sichergestellt werden, dass bei fehlender Adaptation an die neue Situation die Ausgangslage wieder hergestellt werden kann $[12,13]$.

Im Folgenden wird anhand eines Fallbeispiels [14] die klinische Umsetzung dieses auf Evidenz basierenden Therapiekonzeptes der biologischen Antwort dargestellt.

\section{Anamnese}

Eine 59-jährige Patientin stellte sich mit massiven Kiefer-, Gesichts- und Nackenschmerzen sowie in einem psychisch schwer beeinträchtigten Zustand vor. Das ausführliche Gespräch ergab eine mehr als 4 Jahre dauernde zahnärztliche Vorgeschichte, in deren Verlauf unbegründet und aus fehlgeleiteter Ganzheitlichkeit alle Amalgamfüllungen und ein Teil der wurzelbehandelten Zähne entfernt wurden. Während dieser Therapiemassnahmen erfolgte auch eine unkontrollierte Veränderung der Lagebeziehung zwischen Oberund Unterkiefer, die muskuläre Verspannungen im Kopf- und Halsbereich auslöste. Ein Bisshöhenverlust führte bei einer Klasse-II-Verzahnung zudem zum schmerzhaften Einbeissen der Frontzähne des Unterkiefers in die Gaumenschleimhaut. Die funktionelle Orientierungslosigkeit hatte

\section{KARGER}

Fax +497614520714 Information@Karger.d www.karger.com
(๑) 2011 S. Karger GmbH, Freiburg

Accessible online at: www.karger.com/szg
Prof. Dr. med. dent. Christian E. Besimo

Abteilung für Orale Medizin

Aeskulap-Klinik

Gersauerstrasse 8, 6440 Brunnen, Schweiz

christian.besimo@aeskulap.com 
eine massive psychische Belastung der Patientin mit Angstzuständen zur Folge, die die Kiefer-, Gesichtsund Nackenschmerzen zusätzlich verstärkte.

\section{Befund}

Die klinische und radiologische $\mathrm{Be}$ fundaufnahme ergab eine insuffiziente, nicht kaustabile provisorische Versorgung aller Seitenzähne in beiden Kiefern. Die ungenügende Passgenauigkeit und Überkonturierung der Kunststoffkronen und -brücken verhinderte eine adäquate Mundhygiene und führte $\mathrm{zu}$ einer generalisierten Zahnfleischentzündung. Die alio loco durchgeführte endodontische und konservierende Vorbehandlung war grösstenteils insuffizient. Der Einbiss der Frontzähne des Unterkiefers in die Gaumenschleimhaut infolge des iatrogenen Verlusts an Bisshöhe bestätigte sich. Es bestand weder in retraler noch in habitueller Interkuspidation eine definierte zentrische Kieferrelation. Es lag generalisiert eine teilweise stark palpationsdolente Kau-, Halsund Nackenmuskulatur vor. Eine Rekonstruktion der ursprünglichen Bisssituation war anhand der wenigen zur Verfügung stehenden, unvollständigen Unterlagen nicht mehr möglich. Die allgemeinmedizinische Situation war, abgesehen von der beklagten psychischen Belastung und den Angstzuständen, unauffällig.

\section{Diagnose}

Als Diagnose ergab sich eine iatrogene, dysfunktionsbedingte Erkrankung des Kauorgans mit ausgeprägter psychischer Belastung als Folge.

\section{Initiale Schmerz- und Entspannungstherapie}

Die initiale Schmerztherapie erfolgte durch Selbstbeobachtung (Biofeedback) und Selbstmassage der schmerzhaft verspannten Kiefer- und Gesichtsmuskulatur. Die Massage wurde physikalisch durch Wärme unterstützt. Diese Massnahmen wurden interdisziplinär von medizinischer Seite durch Physiotherapie im Bereich der Hals- und Nackenmuskulatur sowie durch psychologische Betreuung der Patientin ergänzt. Auf diese Weise konnte bereits ein gewisser Abbau der Schmerzen und des Leidensdrucks erreicht werden.

\section{Diagnostische Fallanalyse}

Die aktuelle Lagebeziehung zwischen Ober- und Unterkiefer konnte erst nach der initialen Schmerz- und Entspannungstherapie anhand von zwei Modellpaaren festgehalten werden, die in einem Artikulator montiert wurden. Ein Modellpaar wurde unverändert belassen (Abb. 1). Das zweite Modellpaar diente der diagnostischen Fallanalyse. Eine funktionell optimierte Lagebeziehung zwischen Ober- und Unterkiefer wurde durch eine kinesiologisch unterstützte Bisshebung und Aufwachsen der Kauflächen aller Seitenzähne in einer ebenfalls kinesiologisch überprüften «Freedom in centric»-Relation erreicht. Das weitere Einbeissen der Frontzähne des Unterkiefers in die Gaumenschleimhaut konnte auf diese Weise verhindert werden. In der Exzentrik wurde eine bilaterale und anterior-posteriore Front-Eckzahnführung geplant (Abb. 2).

Kinesiologisch unterstützte Okklusionskorrekturen sollten nur bei mehrfacher Reproduzierbarkeit für therapeutische Zwecke genutzt werden und bedürfen in jedem Fall der biologischen Überprüfung durch Schienen oder Langzeitprovisorien [14].

\section{Therapie}

\section{Vorbehandlung}

Eine sofortige Übertragung der im Artikulator erarbeiteten therapeutischen Okklusion in die Mundhöhle mit einer Aufbissschiene oder mit Langzeitprovisorien war vorerst nicht möglich, da die Pfeilerzähne entweder parodontal, endodontisch oder konservierend unzureichend vorversorgt waren und entsprechend noch vorbe-

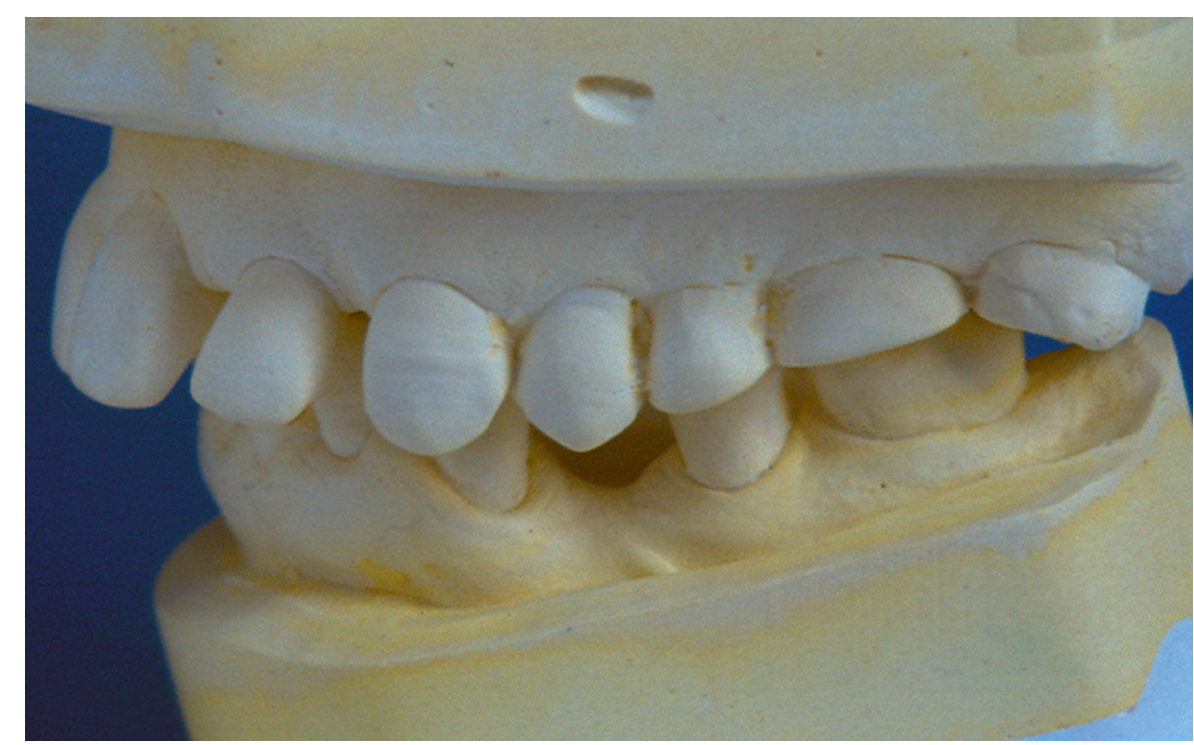

Abb. 1. Studienmodelle der Ausgangssituation, montiert in einem teilweise einstellbaren Artikulator. 


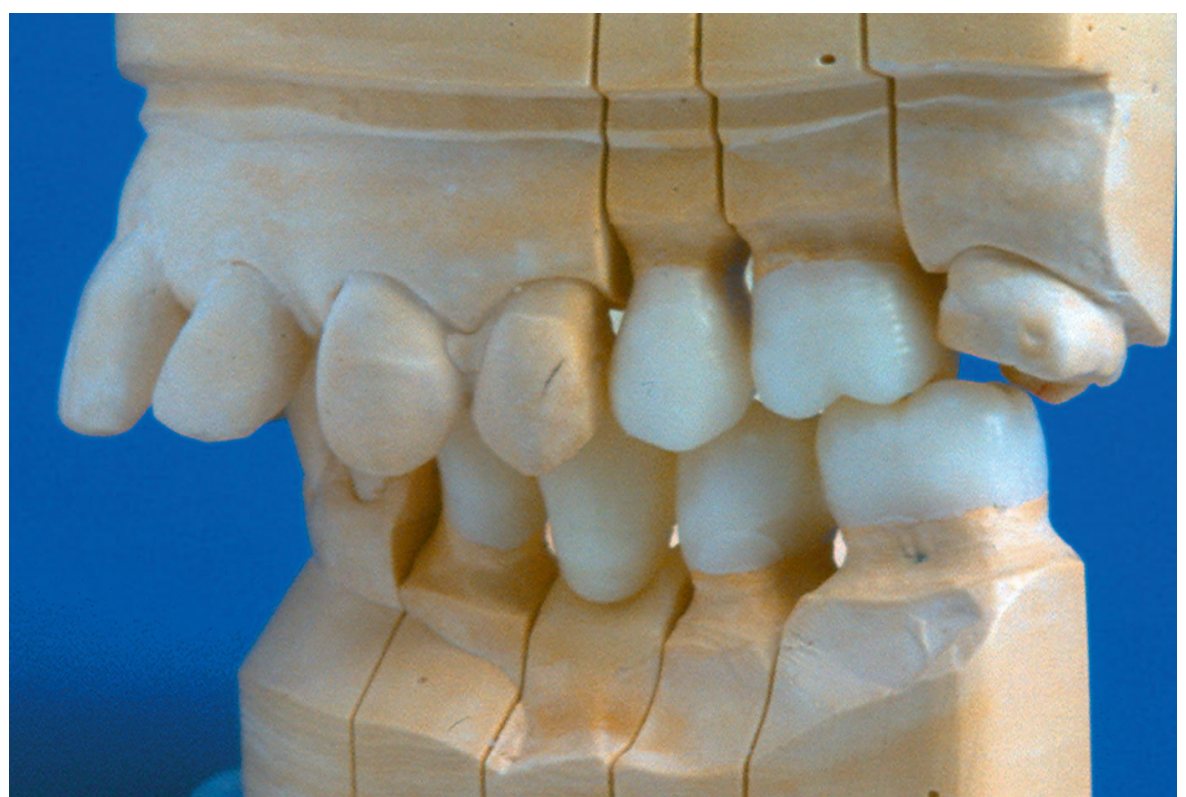

Abb. 2. Diagnostisch aufgewachste therapeutische Okklusion, übertragen auf das Arbeitsmodell für die Herstellung der Langzeitprovisorien.

reitet werden mussten. Infolge von Überkonturierung, abstehenden Rändern und ungenügendem Halt konnte keine der vorbestehenden Kunststoffkronen und -brücken weiterverwendet werden. Sie wurden in einem ersten Schritt kieferweise durch neue, direkt im Mund hergestellte Provisorien in der vorgegebenen Bisslage ersetzt, um die Hygienefähigkeit der Patientin wiederherstellen und die generalisierte schwere Gingivitis behandeln zu können. Zudem erlaubte dieser Therapieschritt die Überprüfung der Compliance.

Von den kurzzeitprovisorisch versorgten Kiefern wurden erneut Modelle angefertigt, die in Relation zum vormontierten Gegenmodell im Artikulator befestigt wurden. Dies erlaubte eine präzise und rückverfolgbare Übertragung der neuen Kieferrelation in den Artikulator. Die bisherige Bisshöhe wurde quadrantenweise mit Kunststoffschlüsseln festgehalten.

Ein besonderes Problem stellten die für die Verankerung von Kronen und Brücken zu kurzen klinischen Kronen dar. Deshalb musste an allen 12 Pfeilerzähnen eine chirurgische Kronenverlängerung durchgeführt werden, um die geplante

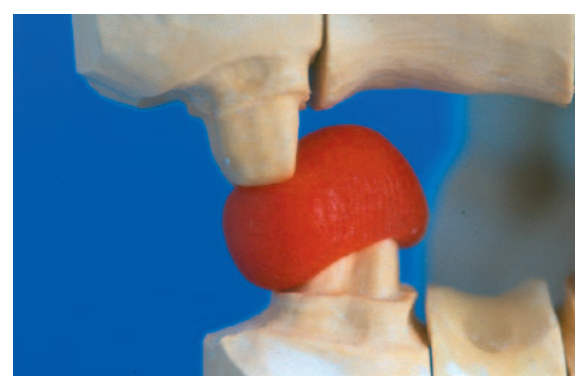

Abb. 3. Im Mund angefertigter Kunststoffschlüssel zur Übertragung der ausgetesteten therapeutischen Bisshöhe in den Artikulator.

rapeutische Okklusion wurde über einen Zeitraum von 10 Monaten an der Patientin ausgetestet. Es erfolgten regelmässige Kontrollen, während deren die Bisshöhe nochmals etwas angehoben und die «Freedom in centric»-Relation leicht erweitert werden musste. Die mit den Langzeitprovisorien erreichte Bisshöhe wurde erneut mit Kunststoffschlüsseln festgehalten. Am Ende dieser intensiven Testphase bestanden stabile und gesunde parodontale Verhältnisse. Die Patientin war nach einer langen Leidenszeit endlich wieder beschwerdefrei. Nun waren die Voraussetzungen für eine definitive prothetische Rehabilitation gegeben. ch Ausheilung des marginalen Parodonts. mit individuellem Löffel und elastomerer Präzisonsabformmasse gewonnenen Arbeitsmodelle wurden erneut mit zentrischen Wachsregistraten zu der jeweils vorbestehenden Situation des Gegenkiefers im Artikulator montiert. Da die Form der Pfeilerzähne durch Präparation wieder verändert wurde, musste die bisherige Bisshöhe nochmals verschlüsselt werden (Abb. 3). Dieses schrittweise Vorgehen, das auch im weiteren Therapieverlauf konsequent beibehalten wurde, erlaubte im Fall einer Verschlechterung des allgemeinen oder lokalen Befindens jederzeit die exakte Rückkehr zu einer der vorbestehenden okklusalen Situationen.

Die mit den langzeitprovisorischen Kronen und Brücken aufgebaute the-

\section{Definitive prothetische Versorgung}

Diese wurde zuerst für den Unterkiefer gegen ein Modell der langzeitprovisorischen Situation des Oberkiefers hergestellt. Form und Funktion der erfolgreich ausgetesteten Langzeitprovisorien wurden exakt übernommen. Nach einer kurzen Tragezeit der definitiven Arbeit im Unterkiefer wurde die Versorgung des Oberkiefers in Angriff genommen. mit Keramikteilkronen, -kronen und -brücken vorgenommen. Die Teilkronen wurden sofort adhäsiv zementiert, die Kronen und Brücken erst nach einer weiteren Testphase von 6 Wochen (Abb. 4). Zum Abschluss wurde eine lediglich nachts zu tragende Aufbissschiene angefertigt, um Die definitive Rehabilitation wurde 

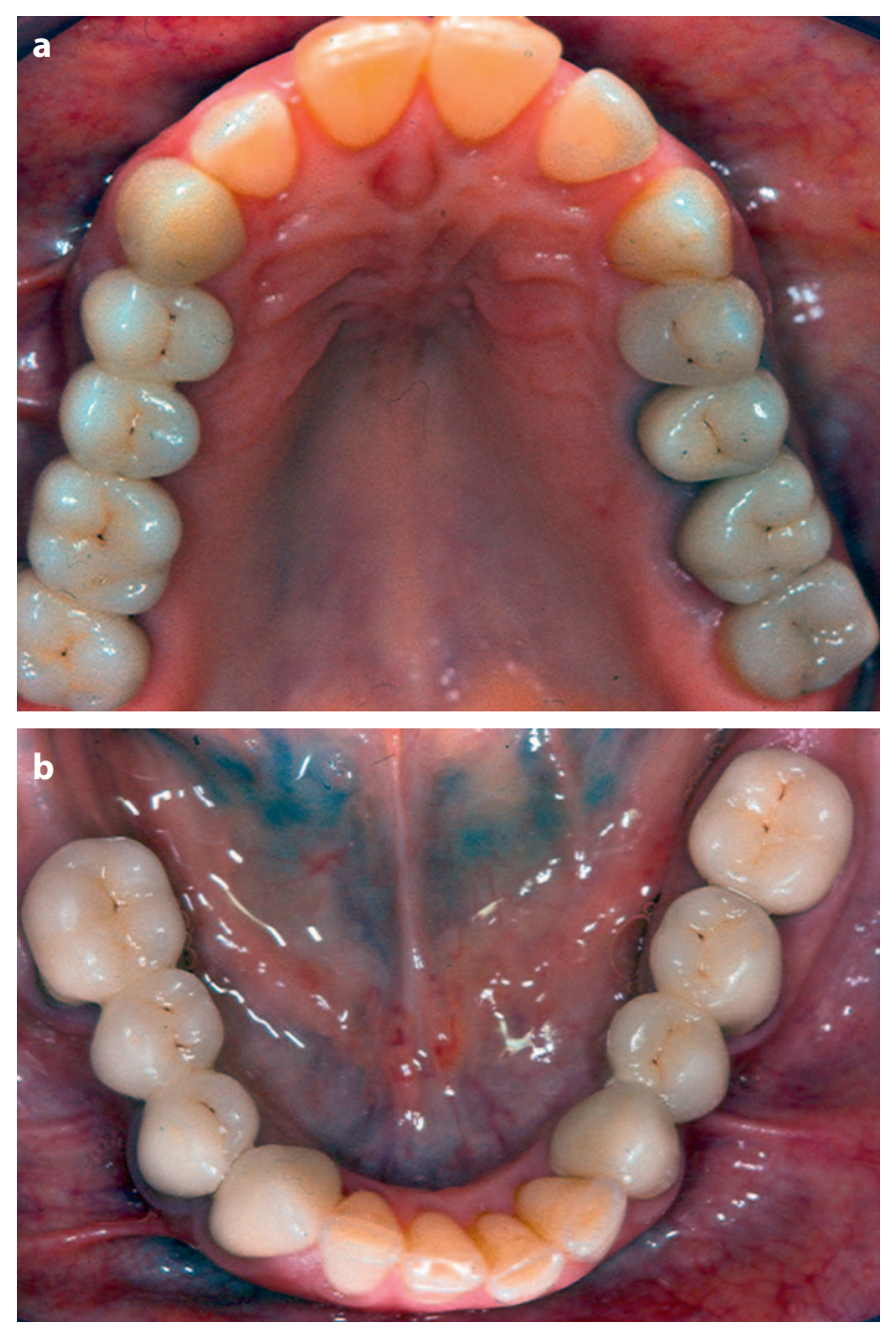

Abb. 4. Klinischer Schlussbefund. a Im Oberkiefer. b Im Unterkiefer.

die in der retralen Kontaktposition nicht abgestützten Schneidezähne des Unterkiefers an einer möglichen Elongation zu hindern.

\section{Diskussion}

Nach einer Behandlungszeit von 2 Jahren und 3 Monaten konnten trotz komplexer Ausgangslage wieder gesunde und stabile orale Verhältnisse sowie als deren Folge auch eine gute psychische Situation für die Patientin erreicht werden [14]. Dieses Behandlungsergebnis konnte mittels regelmässiger jährlicher Kontrollen bis zum heutigen Zeitpunkt über 9 Jahre ohne Komplikationen erhalten werden. Anamnese, Befund und Therahätten vermieden werden können. haupt erst ermöglichten, gemeinsam mit dem Behandlungsteam das schrittweise Herantasten an physiologische funktionelle Verhältnisse während einer langen und aufwendigen Therapie durchzustehen. Die präzise Planung von Art und Abfolge der einzelnen Interventionen gewährleistete nicht nur jederzeit bei Auftreten von Komplikationen eine Reversibilität der okklusalen Veränderungen, sondern vor allem auch die Nutzung der individuellen biologischen Antwort zugunsten einer erhöhten therapeutischen Sicherheit [8].

\section{Literatur}

1 Leon AR: The periodontium and restorative procedures. A critical review. J Oral Rehabil 1977;4:105-117.

-2 Nyman S, Lindhe J: A longitudinal study of combined periodontal and prosthetic treatment of patients with advanced periodontal disease. J Periodontol 1979;50:163-169.

$\checkmark 3$ Greene CS: Managing TMD patients: initial therapy is the key. J Am Dent Assoc 1992;123:43-45.

4 Plesh O, Stohler CS: Prosthetic rehabilitation in temporomandibular disorder and orofacial pain patients. Dent Clin North Am 1992;36:581-589.

5 Besimo CE: Orale und systemische Erkrankungen. Teil 1: Eine auf Evidenz basierende Übersicht ihrer Wechselwirkungen. Schweiz Zschr Ganzheitsmedizin 2008;20:406-411.

6 Besimo CE: Orale und systemische Erkrankungen. Teil 2: Interdisziplinäres Assessment des alternden Menschen. Schweiz Zschr Ganzheitsmedizin 2009;21:54-58.

pieverlauf legten die Annahme nahe, dass bei entsprechend sorgfältiger Planung und Behandlung die alio loco durch ein fehlgeleitetes Verständnis von Ganzheitlichkeit aufgetretenen schwerwiegenden Komplikationen sowie die nachfolgend notwendig gewordene, aufwendige Rehabilitation

Die systematische Diagnostik und Therapieplanung, die ausführliche Aufklärung über jeden Arbeitsgang und das schrittweise, interdisziplinär vernetzte Vorgehen erlaubten es der Patientin, das Vertrauen wiederzugewinnen und allmählich zu einer angstfreien, positiven Lebenseinstellung zurückzufinden $[5,6]$. Dies waren die grundlegenden Voraussetzungen, die es der Patientin über-
7 Lindhe J, Nyman S: Treatment planning; in Lindhe J, Karring T, Lang NP (eds): Clinical Periodontology and Implant Dentistry, ed 3. Copenhagen, Munksgaard, 1997, pp 420-437.

8 Wilson TG: Compliance. A review of the literature with possible applications to periodontics. J Periodontol 1987;58:706-714.

9 Lombardi RE: The principles of visual perception and their clinical application to denture esthetics. J Prosthet Dent 1973;29:358382.

10 Shavell HA: Mastering the art of provisionalization. J Calif Dent Assoc 1979;4:4451.

11 Ferencz JL: Fabrication of provisional crowns and fixed partial dentures utilizing a 'shell' technique. N Y J Dent 1981;51:201-206.

12 Yuodelis RA, Faucher R: Provisional restorations: an integrated approach to periodontics and restorative dentistry. Dent Clin North Am 1980;24:285-303.

13 Bral M: Periodontal considerations for provisional restorations. Dent Clin North Am 1989;33:457-477.

14 Besimo CE, Rohner H-P: Prothetische Rehabilitation bei iatrogenen Funktionsbeschwerden des Kauorgans. Quintessenz 2003;54:263-270. 